\title{
Semiconductor-based Electret Sensors for Sound and Pressure
}

\author{
J. A. Voorthuyzen, P. Bergueld \\ University of Twente, Enschede \\ and A. J. Sprenkels \\ Twente Technology Transfer, Enschede, The \\ Netherlands
}

\begin{abstract}
In this paper the theory and experimental results of integrated electret-based silicon sensors for the detection of sound and pressure are presented. A silicon electret microphone for use in hearing-aid microphones is described, with an experimentally determined sensitivity of $19 \mathrm{mV} / \mathrm{Pa}$ in the frequency range of $50 \mathrm{~Hz}$ to $10 \mathrm{kHz}$. Also the realization of a pressure sensor, developed for application in catheter-tip blood-pressure measurements, is discussed. This type of electret sensor appeared to have a pressure sensitivity of $1.6 \mu \mathrm{V} / \mathrm{Pa}$ in the required frequency range of 0 to $100 \mathrm{~Hz}$.
\end{abstract}

\section{INTRODUCTION}

$\mathrm{M}$ ANY electret-based mechanical sensors are realized by means of conventional construction techniques. Much of the processing is performed manually, which is very labor intensive. Although the process can be highly automated, the manufacturing of these sensors is still piece-wise, and using these techniques, the limits of miniaturization have almost been reached. On the other hand, the application of photolithographic processes and micromachining, which are batch production techniques, is well established nowadays for the construction of three-dimensional sensor structures. The use of these modern techniques has furthermore offered the possibility to integrate sensors that perform a physical to electrical conversion with appropriate electronic circuitry, required for amplification of the sensor output signal and impedance transformation. Another aspect of the use of mono-crystalline silicon wafers is the interesting mechanical property of silicon as such and the possibility to realize predefined three-dimensional structures in it by exploiting the different etching properties for the various crystal axes [1].

Nevertheless, the use of these techniques for the realization of electret-based sensors is rather new, because with the development of integrated electret silicon sensors additional problems are being evoked which have to be solved. Examples of such problems are the micromachining of polymer electrets and the use of other (inorganic) insulators as electrets, of which detailed results are presented elsewhere [2].

After solving these problems we have been able to realize mechanical sensors in silicon, containing electrets. In this paper we present results of developing a sensor for the detection of sound as well as a sensor that can be used for the measurement of pressure.

0018-8367/89/0400-267\$1.00 (C) 1989 IEEE 
In Section 2 the theory of electret-based sensors will be summarized. In Section 3 we present a silicon microphone in which the silicon has been exploited with respect to its micromachining as well as the silicon dioxide $\left(\mathrm{SiO}_{2}\right)$ with respect to its electret properties. In Section 4 we present a silicon pressure sensor in which we have focused on the integration of the electret sensor, based on a Teflon-FEP $\mathrm{PM}^{\mathrm{TM}}$ electret, and the electronic function, resulting in a new type of field effect transistor that is sensitive to pressure. In Section 5 the experimental results will be discussed and in Section 6 some conclusions will be drawn.

\section{THEORY}

A $\mathbf{N}$ electret-based sensor consists basically of a capacitor which is charged due to the presence of an electret between both capacitor plates and which is connected to an electronic circuit which amplifies the signal and or matches the impedance of the device. One of both capacitor plates, usual the one to which the electret is not deposited, is so thin that it can be considered to behave like a so-called diaphragm which deforms as a function of an applied pressure difference across it. The sensor, as schematically drawn in Figure 1, may be modeled by an air-gap capacitance $C_{a}$ in series with an electret capacitance $C_{e}$, which depend on their dimensions as

$$
\begin{gathered}
C_{a}=\frac{\varepsilon_{a} A}{s_{a}-s_{d}} \\
C_{e}=\frac{\varepsilon_{e} A}{s_{e}}
\end{gathered}
$$

with $s_{e}$ the thickness of the electret, $s_{\mathfrak{a}}$ the 'size' of the air-gap as determined by its geometrical design, $s_{d}$ the deflection of the diaphragm due to an external force (pressure, sound, electrostatic force, etc.), and $\varepsilon_{a}$ and $\varepsilon_{e}$ the permittivity of the air-gap and the electret material, respectively. Note that in practical cases the deflection of the diaphragm is not uniform, but that it is maximal at its center and zero at its edge. We assume however a uniform diaphragm deflection $s_{d}$, equal to the center deflection. $A$ is the effective area of the metal electrodes on top of the pressure sensitive diaphragm and the electret. Due to the fact that between air-gap and electret no conductive layer or electrode is present, the effective area $A$ equals the smaller value of the two electrodes.

The electret charge per unit area, which is thought to be located at the electret air-gap interface, is represented as $q_{e}$. The connected amplifier has an input capacitance $C_{i}$ and an input resistance $R_{i}$. Note that

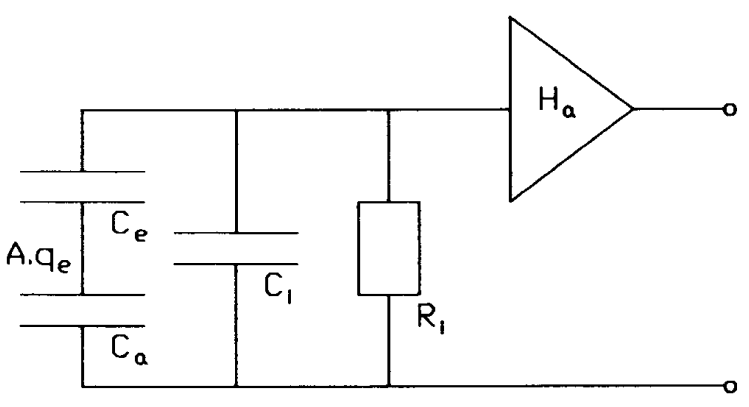

Figure 1.

Schematic drawing of an electret-based mechanical sensor with amplifier.

for a minimal loss of sensor output signal as well as a good frequency response, the amplifier should have a low input capacitance and a high input resistance.

Assume now that a pressure or sound wave $\delta P$ acts on the diaphragm and that it changes the real air-gap space $s_{a}-s_{d}$ with a fraction $\delta s_{d}$. The ratio $\partial s_{d} / \partial P$ is mainly determined by the dimensions, the Young's modulus, and the tension of the diaphragm, and the dimensions of the air-gap. We define this ratio as the mechanical sensitivity $S_{m}$. Assuming a zero initial ténsion in the diaphragm, the mechanical sensitivity at the center of a square diaphragm with side $2 a$ may be approximated by

$$
S_{m}=\frac{\delta s_{d}}{\delta P}=\frac{-1}{\left[\frac{h^{3} E\left[1+1.6\left(s_{d} / h\right)^{2}\right]}{0.138 a^{4}}\right]+\left[\frac{\gamma P_{0}}{s_{0}}\right]}
$$

where $h$ is the thickness and $E$ the Young modulus of the diaphragm material, $s_{d}$ is the center deflection of the diaphragm, $\gamma$ is the specific heat ratio, $P_{o}$ the atmospheric pressure and $s_{o}$ the effective thickness of the air-gap [3]. In case of considering a circular diaphragm with radius $a$, instead of a square with side $2 a$, a similar equation is obtained, in which the constant 1.6 is replaced by 0.5 and the constant 0.138 by 0.170 .

We observe that the mechanical sensitivity is determined by two restoring forces acting on the diaphragm. The first is due to the mechanical properties of the diaphragm, the second due to the compression of the air volume behind the diaphragm.

Due to the presence of the electret charge $q_{e}$, a static electric field $E_{a}$ in the air gap exists. Keeping in mind that it is usual to have conductive electrodes 
with equal size (the capacitor plates) it can be shown that this field equals

$$
E_{a}=\frac{q_{e} s_{c}}{\varepsilon_{a} s_{e}+\varepsilon_{e}\left(s_{a}-s_{d}\right)}
$$

This electric field is accompanied by an electrostatic force or pressure which acts on the diaphragm. This pressure causes a static diaphragm deflection and thus contributes to the actual value of $s_{d}$. It can be shown that the electrostatic pressure $P_{e}$ can be written as

$$
P_{e}=\frac{E_{a}^{2}}{2 \varepsilon_{a}}
$$

Considering Equations (3)-(5) it will be clear that the calculation of the actual value of $P_{e}$ and the corresponding value of $s_{d}$ is rather complex, because both parameters depend on each other and are non-uniform over the whole diaphragm. As a first approximation we therefore will use in the succeeding part of our paper the value of $P_{e}$, corresponding to $s_{d}=0$, and calculate an average value of $s_{d}$ by using. this value of $P_{e}$ and using Equation (3).

The open-circuit electrical sensitivity $S_{\epsilon}$, defined as the quotient of the voltage, present between the input nodes of the amplifier in the case it has an infinite input impedance $\left(R_{i}=\infty\right.$ and $\left.C_{i}=0\right)$ and the diaphragm displacement $\delta s_{d}$, is equal to the value of the electric field $E_{a}$ in the air-gap.

Usually an amplifying circuit has a finite input impedance, which causes the voltage at its input nodes to be lower that the open-circuit voltage of the electretbased sensor. For diaphragm displacements $\delta s_{d}$, which are fast compared to the time constant $\tau$, (determined by $R_{i}, C_{a}, C_{e}$ and $C_{i}$ ), the transfer function $H_{c}$ from open-circuit voltage to amplifier input voltage is due to a mismatch of the different capacitances only, and can be written as

$$
H_{c}=\frac{C_{m}}{C_{m}+C_{i}}
$$

Where

$$
C_{m}=\frac{C_{a} C_{e}}{C_{n}+C_{e}}
$$

If the amplifier transfer from input to output is written as $H_{a}$, the overall sensitivity $S$, defined as the quotient of amplifier output and sensor input, usually a voltage or current and (sound) pressure respectively, can be denoted as

$$
S=S_{m} S_{e} H_{c} H_{a}
$$

In the following Sections we will show in which way actual sensors can be designed as well as developed, considering the basic theory given in this Section. We will consider an electret microphone with a required frequency range of $50 \mathrm{~Hz}$ to $10 \mathrm{kHz}$ and a minimum detectable sound pressure of $4 \times 10^{-4} \mathrm{~Pa}$., as well as a pressure sensor with a desired frequency range of 0 to $100 \mathrm{~Hz}$. and a minimum detectable pressure of $10 \mathrm{~Pa}$. For the microphone it appears that we have to pay considerable attention to its overall sensitivity, while for the pressure sensor the required sensitivity to static pressures appears to be a serious source of concern. The differences in the required performances of both sensor types yield two sensor realizations that are quite different from each other.

\section{SILICON MICROPHONE}

\subsection{DESIGN CONSIDERATIONS}

T OR a microphone, e.g. used in hearing-aid applications, the minimum sound pressure level to be detected is rather low, viz. $4 \times 10^{-4} \mathrm{~Pa}\left(4 \times 10^{-3} \mu\right.$ bar $)$, while the maximum detectable sound pressure level is $20 \mathrm{~Pa}$, with a required frequency range of approximately $50 \mathrm{~Hz}$ to $10 \mathrm{kHz}$. This large dynamic range of $95 \mathrm{~dB}$ forces the designer to consider and optimize the microphone sensitivity.

Considering the required sensitivity and the theory presented in Section 2 we have concluded the following:

1. The sensor capacitance should be large compared to the amplifier input capacitance, which can be accomplished by choosing a large value of the electrode area $A$.

2. The electret charge should be as large as possible, to yield a high value of the electrical sensitivity. Optimization of the electrical sensitivity, which is performed by means of the electret charge may have considerable influence on other quantities determining the overall sensitivity of a sensor. A larger value of the electret charge causes a larger electrostatic pressure (Equation (5)) and thus also a larger value of $s_{d}$ (Equation (3)). A larger value of $s_{d}$ results in a lower value of the mechanical sensitivity $S_{m}$ (Equation (3)) and a higher value of $H_{c}$, as can ben seen from Equations (1), (6), and (7). Especially for larger values of the electret charge it is difficult to predict the influence of an increased electret charge on the overall sensor sensitivity $S$. In certain cases even a reduction of sensitivity has been observed for increasing values of the electret 
charge. Note that the maximum value of the electret charge is also limited by the fact that the static diaphragm deflection $s_{d}$ should of course be smaller than the original air-gap 'size' $s_{a}$.

3. Mechanical sensitivity also should be optimized. Using Equation (3) we see that it increases for larger and thinner diaphragms. However, one has to realize that in that case also the static deflection due to the electrostatic force increases, limiting the possible maximum and minimum values of diaphragm size and thickness respectively.

4. The second term in Equation (3) that influences the mechanical sensitivity is due to the compression of air in the air-gap, causing an increasing pressure in it which counteracts the acoustic pressure. Due to the fact that the static pressure in the air-gap always equals the barometric pressure of the environment via one or more small holes, the term $P_{o} / s_{o}$ only influences the dynamic and not the static mechanical sensitivity. This implies that its influence should be as small as possible, which can be realized by adding to the microphone a backchamber which is connected to the microphone air-gap via some small holes in the electret-covered capacitorplate, as shown in Figure 2.

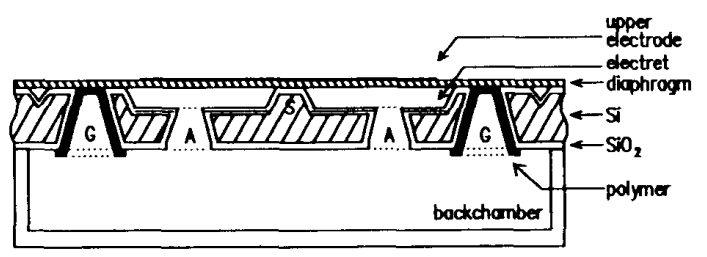

Figure 2.

Cross-section of an electret microphone.

We decided to develop a microphone which comprises sixteen diaphragm supports. These supports, of which one is indicated as $S$ in Figure 2, are necessary to prevent the diaphragm from collapsing towards the back plate due to the electrostatic attraction mentioned above. The microphone can thus be considered to be composed of nine small unit cells, or small microphones, operating in parallel. The dimensions of a unit cell determine the above mentioned open-circuit electrical sensitivity as well as the frequency response. That several unit cells are connected in parallel is to obtain a microphone capacitance which is large enough as compared to the amplifier input capacitance, avoiding a too large capacitive attenuation, as defined by Equation (6). In this way the sensitivity factors $S_{m}$ and $H_{c}$ can be optimized independently of each other.
For our microphone design we have chosen the following materials and dimensions.

1. As an electret we have used $\mathrm{SiO}_{2}$ with a thickness $s_{c}=1.1 \mu \mathrm{m}$, as described elsewhere [2]. The permittivity $\varepsilon_{e}=34.5 \times 10^{-14} \mathrm{~F} / \mathrm{cm}$. In the initial stage of our research we have applied Teflon-FEP electrets. However, in a later stage we have found that under certain conditions $\mathrm{SiO}_{2}$ can also be used as an electret [2]. Due to the fact that the use of $\mathrm{SiO}_{2}$ in silicon-based sensors is less complicated than the use of Teflon-FEP, we have afterwards focused on microphones with $\mathrm{SiO}_{2}$ electrets.

2. The air-gap is filled with air, which has a permittivity $\varepsilon_{a}=8.85 \times 10^{-14} \mathrm{~F} / \mathrm{cm}$.

3. The back-chamber distance $s_{o}$ of the microphone is chosen to be $2 \mathrm{~mm}$, and the specific heat ratio $\gamma=$ 1.67 .

4. As the diaphragm material we have applied oneside metallized Mylar ${ }^{\mathrm{TM}}$ foils, which have a Young's modulus $E=5 \times 10^{9} \mathrm{~N} / \mathrm{m}^{2}$, with a thickness $h=2.5 \mu \mathrm{m}$.

5. Due to the fact that the microphone is designed for use in hearing aids we have chosen a square diaphragm with outer dimensions of $2.45 \times 2.45 \mathrm{~mm}^{2}$. Realizing that we have used diaphragm supports, as drawn in Figure 5 and 6 , this implies that we obtain nine smaller square diaphragms with a side $2 a=0.82 \mathrm{~mm}$.

6. Assuming the whole Mylar diaphragm to be metallized this implies that the value of the electrode area $A$, determining the capacitances $C_{a}$ and $C_{e}$ is $A=6$ $\mathrm{mm}^{2}$.

7. The initial air gap $s_{a}$, as determined by the microphone geometry only, has been chosen $20 \mu \mathrm{m}$.

8. As the amplifier we have used a JFET sourcefollower configuration with an estimated transfer function $H_{a}=0.8$, and an effective input capacitance of $1 \mathrm{pF}$.

Using the model of Section 2, we calculated in a computer simulation the optimum value of the electret charge. The results are shown in Figure 3. We observe that the sensitivity increases for increasing negative values of the electret voltage $V_{e}=A q_{e} / C_{e}$, until a value of about $-300 \mathrm{~V}$ is reached. For higher voltages a slow decrease is observed, due to the interaction between mechanical and electrical sensitivity, as mentioned above. 


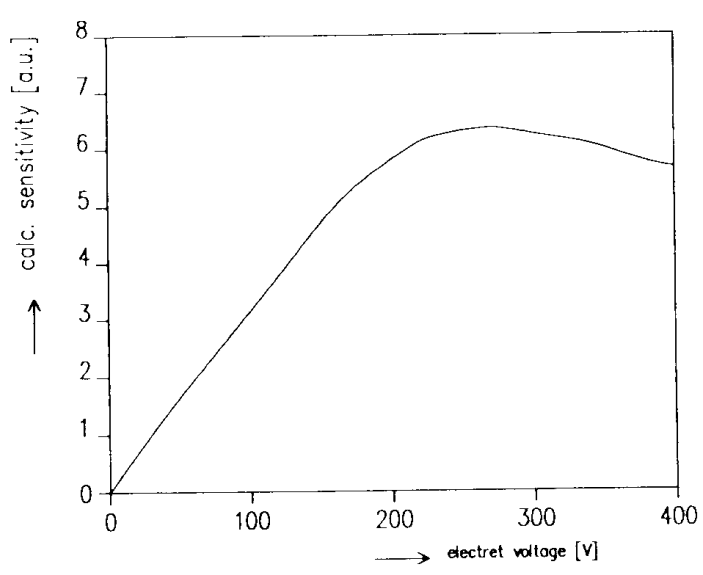

Figure 3.

Calculated normalized overall microphone sensitivity as a function of electret voltage.

In our microphone design we have chosen an electret voltage of $-325 \mathrm{~V}$.

We have calculated that an electret voltage of $-325 \mathrm{~V}$ causes a static diaphragm deflection $s_{d}=4.5 \mu \mathrm{m}$. Using now the Equations (1), (2), (6) and (7) we have calculated that the transfer function $H_{c}=0.74$, and that the amplifier-input voltage sensitivity is about $23 \mathrm{mV} / \mathrm{Pa}$.

\subsection{MICROPHONE REALIZATION}

For the realization of the silicon back plate we start with a n-type 5 to $10 \Omega \mathrm{cm}$ (100)-oriented oxidized silicon wafer. A $1 \mu \mathrm{m}$ thick oxide layer is used as a mask for the KOH solution which has been used as the anisotropic etchant (Figure 4a). First the air cavities are etched to a depth of $20 \mu \mathrm{m}$. This is performed by patterning the $\mathrm{SiO}_{2}$ layer and subsequently etching the wafer in a $\mathrm{KOH}$ solution (Figure 4b).

After subsequent oxidation (Figure 4c) both sides of the wafer are patterned and etched simultaneously using $\mathrm{KOH}$ as the anisotropic etchant. On the reverse side of the wafer two types of holes are etched through the wafer. One type (A) ends in the air cavity and forms the necessary connection between the air gap and the back chamber behind the back plate as shown in Figure 2. The other type $(G)$ is etched around the air cavity area, in order to be able to attach the diaphragm to the back plate in a later processing step. At the same time $\mathrm{V}$-grooves are etched around the structures. This
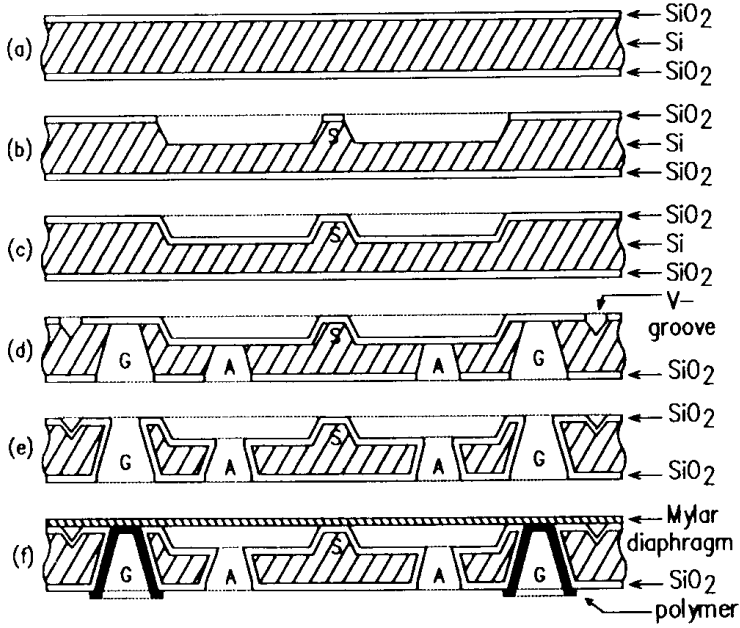

Figure 4

Processing steps of the silicon electret microphone.

simplifies the dicing of the individual microphones after the processing has been completed. The result of this anisotropic etching process is shown in Figure 4d.

After etching the remaining $\mathrm{SiO}_{2}$, the wafer is thermally oxidized again for about $3 \mathrm{~h}$ at $1150^{\circ} \mathrm{C}$, resulting in a $1.1 \mu \mathrm{m}$ thick $\mathrm{SiO}_{2}$ layer on the entire wafer (Figure $4 \mathrm{e}$ ). Next the $\mathrm{SiO}_{2}$ surface is chemically treated in order to reduce the lateral surface conduction $[2,3]$. Subsequently the $\mathrm{SiO}_{2}$ layer is charged to $-325 \mathrm{~V}$ using a corona charging method which has proven to be an adequate method to charge a complete wafer. At this stage of the process the diaphragm has to be attached. This is done by the fixation of a $2.5 \mu \mathrm{m}$ thick Mylar $^{\text {IM }}$ foil on top of the complete wafer. The actual attachment results from spraying an adhesive polymer through the holes $(G)$ around the air cavities. This method yields a strong attachment of the diaphragm on the back plate. The resulting cross section is shown in Figure 4f. Next the upper electrode is evaporated on top of the diaphragm. Finally the wafer can be diced, resulting in separate microphones.

A top-view of the microphone is to be seen in Figure 5 . Note that the cross-sectional view of Figure 4 has been taken along the dashed curve B-B'.

\subsection{EXPERIMENTAL RESULTS}

The sensitivity as well as the frequency response of the silicon electret microphones has been measured 


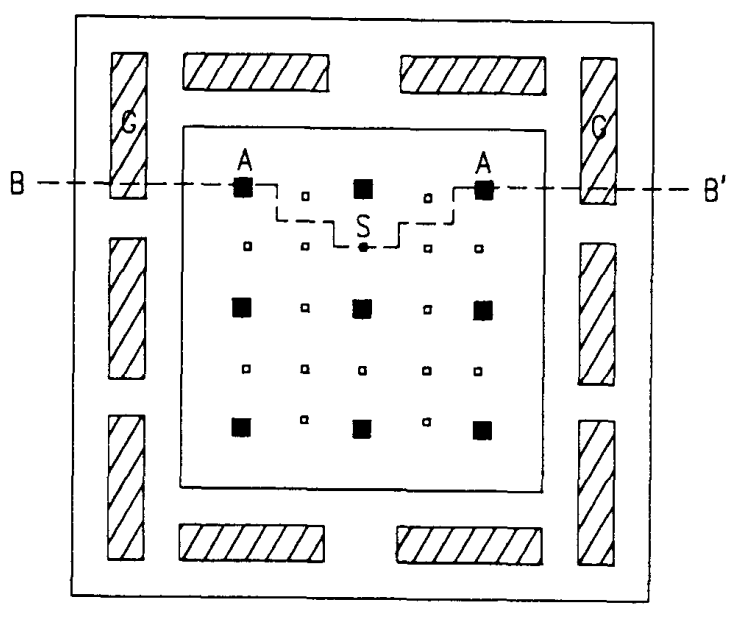

Figure 5 .

Top-view of the silicon electret microphone before diaphragm attachment.

by connecting the electret sensor to a JFET source follower amplifier with an input capacitance $C_{i}=1 \mathrm{pF}$, and applying a standard 'artificial voice' (B\&K 4219) as the acoustic reference source. The frequency range of our measurement setup is limited from $100 \mathrm{~Hz}$ to about $15 \mathrm{kHz}$. The result is presented in Figure 6 . The amplifier-input voltage sensitivity, determined by measuring the amplifier output voltage and using the experimental value of $H_{a}$, is about $19 \mathrm{mV} / \mathrm{Pa}$.

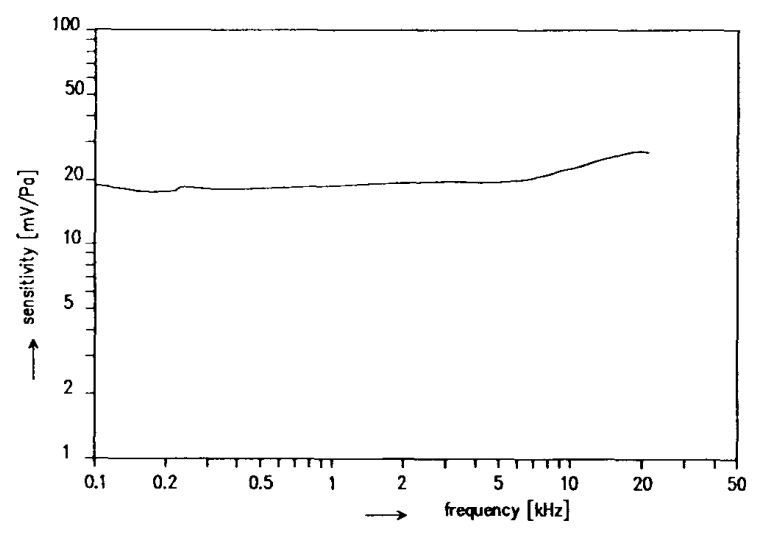

Figure 6.

The measured frequency response of the prototype silicon microphone, as referred to the amplifier input node.

The curve in Figure 6 is a typical example of one of numerous microphones which have been tested. We have found that the sensitivity of microphones, manufactured in one silicon wafer, differs less than $5 \%$ from the average value.

In addition to this design, as described in Section 3.1, other microphones with quite different geometries (more or less diaphragm supports, thinner air-gap, thinner as well as thicker diaphragms etc.. ) have been developed and tested, in order to further optimize the microphone performance. In this way microphones with an opencircuit sensitivity of $250 \mathrm{mV} / \mathrm{Pa}$ have been realized.

An interesting aspect is that by means of this research we have found that, while keeping all other parameters the same, the use of a patterned electrode on top of the Mylar diaphragm may increase the overall sensitivity of the microphone. We therefore have realized a shadow mask which is placed on the Mylar diaphragm during the evaporation of the thin metal layer, which is used as one of the capacitor electrodes, on top of the diaphragm. Using this mask, only the central part of each unit cell is provided with an electrode, and these electrodes are connected to each other by means of very small metal strips. We have measured an increase of the overall sensitivity of about $6 \mathrm{~dB}$ (a factor 2 ) if we use electrodes with a $40 \%$ reduced size, located at the center of the diaphragm of each unit cell. We have found also that for smaller electrodes the overall sensitivity starts to decrease again. The reason for this fact is that on the one hand the transfer function $H_{c}$ decreases for lower values of $A$ due to the lower values for the capacitances $C_{a}$ and $C_{e}$, but that on the other hand the average mechanical sensitivity increases, because the deflection at the diaphragm center is maximal, while it is zero at the diaphragm edges.

\section{PRESSURE SENSOR}

\subsection{DESIGN CONSIDERATIONS}

Enerally, pressure sensors can be considered to $G$ be divided in two categories, namely absolute and differential pressure sensors. We have focused on the realization of a differential pressure sensor, suitable for the measurement of static and low-frequency pressures from about 10 to $2 \times 10^{4} \mathrm{~Pa}$ in the frequency range: 0 to $100 \mathrm{~Hz}$. for application in catheter tip blood pressure measurements.

Considering the required sensitivity and dynamic range of this pressure sensor we have concluded the following: 
1. It is to be expected that the sensitivity of this pressure sensor will not be a serious problem, if we keep in mind the required sensitivity for a microphone, as mentioned in Section 3.1 .

2. The essential difference between both sensors is that for a pressure sensor the input resistance $R_{i}$ of the amplifier as drawn in Figure 1 has to be infinite to exclude any time-decay of the overall sensor output signal. However, on the contrary for most amplifiers a finite value of the input resistance is necessary for two reasons. The first is to bias the amplifier input stage to provide the small input bias current associated with the use of a JFET (junction field effect transistor) as the active component. The second reason is to eliminate the influence of disturbing leakage currents flowing to or from the amplifier input terminal and to reduce the influence of electrostatic fields as well as charges by forcing the dc potential of the node between electret sensor and amplifier to be constant.

It will be clear that if we want to realize electretbased sensors that are suitable for the detection of static pressures, the amplifier should be modified.

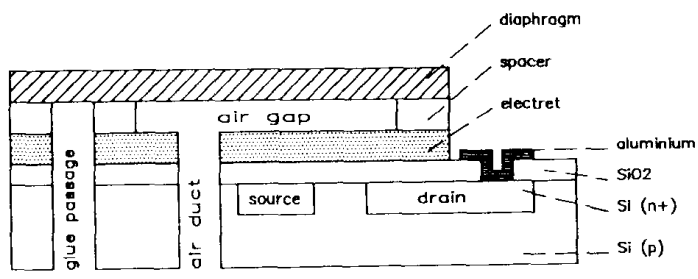

Figure 7 .

Schematic drawing of the integrated electret pressure sensor.

The first thing we can do is replacing the JFET in the input stage of the amplifier by a MOSFET (metal oxide silicon field effect transistor), because this type of transistor does not require an input bias current. Note that in many applications a JFET is preferable due to its lower noise level as compared to MOSFET's. In our application for relatively large pressure, however, the noise will not be a problem. The second step in avoiding a biasing input resistance is the integration of MOSFET and electret sensor, in such a way that the node between electret sensor and amplifier is shielded from external electrostatic charges and fields. An elegant solution is the structure drawn in Figure 7.

We see a conductive, pressure-sensitive diaphragm, separated from the electret by a small spacer, which thickness determines the thickness of the air gap. The electret itself is directly deposited on top of the MOSFET structure. The region between the source and drain, usually called the channel of the transistor, is the active region. The current in the MOSFET, which flows at the $\mathrm{Si} / \mathrm{SiO}_{2}$ interface through this channel from drain to source, depends on the voltage across the $\mathrm{SiO}_{2}$ above the channel.

Note that the integration has been followed out so far that the electrical connection between electret sensor and amplifier, as drawn in Figure 1, can now be made by a thin conductive layer between electret and $\mathrm{SiO}_{2}$. Due to the fact however that any connection to this conductive layer is unwanted for the required sensitivity to static pressures, it seems very attractive to omit this layer, avoiding the impact of electrostatic fields and charges, and reducing the occurrence of leakage currents. This is indeed the case for the sensor, as drawn in Figure 7. Of course this means that the sensitivity will be lower than for the microphone, because the transfer function $H_{c}$ will obtain a much lower value.

This sensor may be regarded as a new type of FET device, which we have called the PRESSFET [5].

In addition to these considerations we have chosen the following materials and parameters that determine the overall sensitivity:

1. As electret we have used electron-beam charged Teflon-FEP with a thickness of $12.5 \mu \mathrm{m}$. The permittivity $\varepsilon_{e}$ of Teflon-FEP is $\simeq 17.7 \times 10^{-14} \mathrm{~F} / \mathrm{cm}$. The reason that we have used in this case Teflon-FEP as electret instead of $\mathrm{SiO}_{2}$, is due to the fact that the PRESSFET research has been initiated long before we started to investigate the electret properties of $\mathrm{SiO}_{2}$.

2. The air-gap is filled with air, which has a permittivity $\varepsilon_{a}=8.85 \times 10^{-14} \mathrm{~F} / \mathrm{cm}$.

3. We decided to design the PRESSFET as a differential pressure sensor, which means that the air in the air-gap equals the barometric pressure. The air will thus not be compressed, which implies that the value of $s_{o}=\infty$ and that the value of $\gamma$ is no longer important.

4. As diaphragm material we have applied aluminum foils, for which Young's modulus $E=10^{11} \mathrm{~N} / \mathrm{m}^{2}$, with a thickness $h=3 \mu \mathrm{m}$.

5. The PRESSFET has been designed for use in the tip of catheters with an outer diameter of 2 to $3 \mathrm{~mm}$. 
Therefore we have chosen a circular diaphragm with a radius $a=250 \mu \mathrm{m}$.

6. Considering the required pressure range of $20 \mathrm{kPa}$ and the mechanical sensitivity as given in Equation (3) we calculated that the thickness of the air-gap should be about $3 \mu \mathrm{m}$.

7. The absence of the metal layer between electret and amplifier input (the conductor between Teflon-FEP and $\mathrm{SiO}_{2}$ ) implies that the active electrode area $A$ determining the values of $C_{a}$ and $C_{e}$ now equals the extremely small size of the channel. Considering the dimensions of the channel it can be calculated that $A=12 \times 10^{3} \mu \mathrm{m}^{2}$.

8. The electret voltage has been chosen $-300 \mathrm{~V}$.

9. Due to the use of a much stiffer diaphragm, the electrostatic deflection $s_{d}$ is ignorably small and has been assumed to be zero.

10. The transfer function $H_{c}$ is determined by the capacitances of the air-gap, the Teflon-FEP layer and the $\mathrm{SiO}_{2}$ layer above the channel, which behaves as the input capacitance. The estimated value of the input capacitance $C_{i}=1.3 \mathrm{pF}$.

11. Using Equations (1), (2), (6) and (7) the transfer function $H_{c}=0.002$.

In order to compare the experimental results of the pressure sensor with those of the microphone we assume that there is a conductive layer between Teflon-FEP and $\mathrm{SiO}_{2}$, and consider this layer as the input terminal of the amplifier. The amplifier transfer function $H_{a}$ from this terminal to the drain-source current is in that case $1.3 \mathrm{~mA} / \mathrm{V}$.

Using these numerical values and the model of Section 2 we have calculated an amplifier input-voltage sensitivity $S_{m} S_{e} H_{c}=8 \mu \mathrm{V} / \mathrm{Pa}$, and an overall sensitivity $S=10 \mathrm{nA} / \mathrm{Pa}$.

\subsection{PRESSURE SENSOR REALIZATION}

We use a process that is quite similar to that discussed for the microphone, except the integrated realization of the electret and the FET.

We apply a CVD (chemical vapor deposition) process to form doped and undoped silicon dioxide layers, which are patterned and then used as diffusion sources to dope the silicon wafer. We start from p-type silicon wafers with an initial boron concentration of about $2 \times 10^{15} \mathrm{~cm}^{-3}$ and a resistivity of about $10 \Omega \mathrm{cm}$. The front and back of the wafer are provided with a borondoped and an undoped oxide layer respectively. The boron-doped layer is used to enhance the boron doping of the wafer, avoiding the occurrence of parasitic channels. The undoped layer on the back is used as an etching mask for the realization of holes through the silicon wafer by means of anisotropic etching from back to front in a later stage of the process.

On the patterned boron doped layer a phosphorusdoped oxide layer is deposited to dope the source and drain regions, and patterned as schematically drawn in Figure 8a.

a)

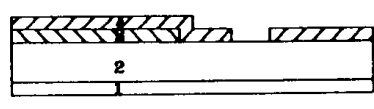

b)

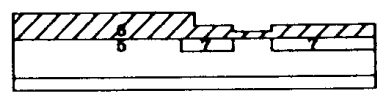

$$
\begin{aligned}
& 1=\mathrm{SiO} 2 \\
& 2=\mathrm{p}^{-\mathrm{Si}} \\
& 3=\mathbf{p}^{+-S i O 2} \\
& 4=\mathrm{n}^{+-\mathrm{SiO} 2} \\
& 5=\mathrm{p}^{+-\mathrm{Si}} \\
& 6=\mathrm{SiO} \\
& 7=\mathrm{n}^{+-S i}
\end{aligned}
$$

c)

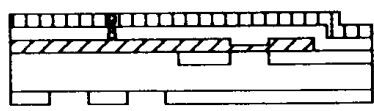

8 = aluminium

$9=\mathrm{CVD}-\mathrm{SiO} 2$

d)

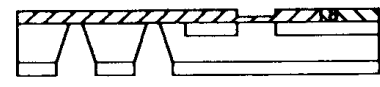

8 = aluminium

e)

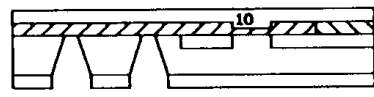

$10=$ TEFLON-FEP

f)

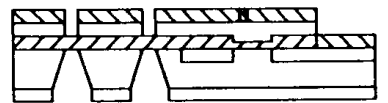

11 = aluminium

g)

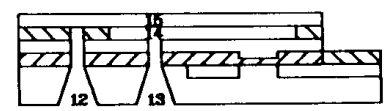

$$
\begin{aligned}
& 12=\text { glue hole } \\
& 13=\text { air duct } \\
& 14=\text { air-gap } \\
& 15=\text { diaphragm }
\end{aligned}
$$

Figure 8.

Processing steps of the electret pressure sensor.

Oxidation of the gate-region and diffusion of boron and phosphorus from the oxide into the silicon are performed at $1150^{\circ} \mathrm{C}$, resulting in the structure drawn in Figure 8b. Now the oxide on the back, which will be used as a mask for etching of holes through the silicon, is patterned and the contact holes on the front, for the 
source, bulk and drain contacts are etched. This step is followed by a $1 \mu \mathrm{m}$ thick aluminum layer evaporated on the front, for the realization of contacts. This aluminum layer is covered with an $\mathrm{CVD}-\mathrm{SiO}_{2}$ layer, to protect the front of the wafer during the etching of holes through the silicon, as schematically drawn in Figure 8c.

Afterwards, the anisotropic etching of holes through the silicon is performed, requiring an etch time of about $8 \mathrm{hr}$, followed by the removal of the oxide layer which is on top of the aluminum. The aluminum layer itself is patterned in order to make the contacts, yielding the structure of Figure 8d.

As electret we apply $12.5 \mu \mathrm{m}$ thick Teflon-FEP foils, which are deposited by a heat-sealing process, as described elsewhere [2]. The resulting structure is drawn in Figure 8e. The Teflon layer is patterned, e.g. above the source, bulk and drain contacts, using a plasma-etch process, masking the parts of the wafer that should not be etched by a $1 \mu \mathrm{m}$ thick aluminum layer [2]. In Figure $8 \mathrm{f}$ we have drawn the structure after etching the Teflon.

The aluminum mask is removed, except for a ring around the gate (the region between source and drain) of the NMOS structure. In this way we can realize an air gap between Teflon and diaphragm, whose zero-pressure space is thus determined by the thickness of the original aluminum mask.

Next the holes through the wafer, which are still closed by a thin oxide layer, as drawn in Figure $8 \mathrm{f}$, are opened by wet chemical etching. As a result we obtain the structure as schematically drawn in Figure $8 \mathrm{~g}$.

The Teflon is charged by means of an scanning electron microscope set-up $[3,5]$ to a charge level that corresponds to an electret voltage of $-300 \mathrm{~V}$. Finally, a $3 \mu \mathrm{m}$ thick diaphragm is attached and bonded to the electret MOS structure by filling the holes of every device, made for that purpose and indicated as glue passage in the Figures 7 and 8 . Note that one hole is not filled with glue because it is used as a reference pressure inlet. This reference pressure is usually the barometric pressure, resulting in a differential pressure relative to the barometric pressure.

\subsection{EXPERIMENTAL RESULTS}

To measure our devices we have realized a closedloop controlled pressure actuator which can generate pressures between $-15 \mathrm{kPa}$ and $+60 \mathrm{kPa}$ relative to the atmospheric pressure.

We have measured an overall sensitivity of $2 \mathrm{nA} \mathrm{Pa}$ over the mentioned pressure range. Using the experimentally determined value of the transfer function $H_{a}$ of the amplifier, which appeared to equal the theoretical value rather well, this corresponds to an amplifier-input voltage of $1.6 \mu \mathrm{V} / \mathrm{Pa}$.

The pressure sensitivity appeared to be constant in the frequency range 0 to $100 \mathrm{~Hz}$.

The sensors showed a rather large sensitivity to variations in the ambient temperature of about $7 \mathrm{mV} /{ }^{\circ} \mathrm{C}$ referred to the theoretical amplifier input node. Considering the pressure sensitivity of $1.6 \mu \mathrm{V} / \mathrm{Pa}$, a change in temperature of $1^{\circ} \mathrm{C}$ has the same result as a pressure change of $4.3 \mathrm{kPa}$.

\section{DISCUSSION}

$T$ $\mathrm{HE}$ calculated microphone sensitivity is $23 \mathrm{mV} / \mathrm{Pa}$, which corresponds rather well to the measured value of $19 \mathrm{mV} / \mathrm{Pa}$. The frequency response appeared to be sufficient for microphone applications.

The calculated sensitivity of the pressure sensor is $8 \mu \mathrm{V} / \mathrm{Pa}$ and the measured value only $1.6 \mu \mathrm{V} / \mathrm{Pa}$, which is a considerable difference. Other experiments have shown that this is mainly due to a much lower value of the mechanical sensitivity than as calculated from Equation (3).

Comparing the measured sensitivity of the microphone $(19 \mathrm{mV} / \mathrm{Pa})$ and the pressure sensor $(1.6 \mu \mathrm{V} / \mathrm{Pa})$ as well as the theoretical values calculated for both we can conclude that this large difference is mainly due to the considerable difference in the value of the parameters $H_{c}$ and $S_{m}$.

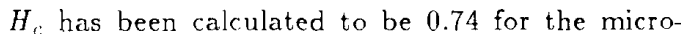
phone and 0.002 for the pressure sensor. This large difference is due to the fact that the size of the electrode area $A$ determining $C_{n}$ and $C_{n}$, equals the small size of the channel area of the MOSFET structure, because the metal layer between Teflon and $\mathrm{SiO}_{2}$ has been omitted in order to prevent the occurrence of leakage currents to it. The second reason for the lower value of the sensitivity is caused by the use of aluminum foils instead of Mylar foils. The Young modulus of aluminum is about 
20 times higher. Assuming all dimensions to be the same for the microphone and the pressure sensor, and using Equation (3) we see that this implies a value of $S_{m}$ that is 20 times lower.

The observed large temperature sensitivity of the pressure sensors is due to the temperature dependence of the FET, which can be approximated to be about $6.5 \mathrm{mV} /{ }^{\circ} \mathrm{C}$ as related to the theoretical amplifier input node. The temperature sensitivity of $4.3 \mathrm{kPa} /{ }^{\circ} \mathrm{C}$ is too high, because we designed this device for the measurement of $10 \mathrm{~Pa}$ or more, which requires that the environmental air should be constant within $0.0023^{\circ} \mathrm{C}$.

Note that in case of the electret microphone the temperature sensitivity is of no importance, because temperature variations occur at frequencies far below the required frequency range for usual microphone application. In case of a device sensitive to static pressures, the temperature variations and the pressure variations are in the same frequency region.

A compensation circuit, added to the pressure sensor, may reduce the temperature sensitivity, but we do not expect that this will be sufficient. Therefore we conclude that an increased pressure sensitivity is necessary for a good pressure sensor.

The increase of the pressure sensitivity can be accomplished by an increased value of $S_{m}$ or of $H_{c}$. A higher value of $S_{m}, e . g$. by replacing the aluminum as diaphragm material by another material, is not possible, because a higher value of the mechanical sensitivity implies a reduced pressure range, and $S_{m}$ has been designed with respect to the maximum pressure to be detected. A higher value of $H_{c}$ can be realized by adding metal between Teflon and $\mathrm{SiO}_{2}$. As already mentioned, we expect that this will result in a leakage current to this electrode, disturbing the low-frequency behavior of the pressure sensor.

Considering the fact that both methods, discussed with respect to an increase of the overall sensitivity of the pressure sensor, are not useful, as well as the much larger influence of disturbing external parameters such as the environmental temperature, we conclude that the present realization of the PRESSFET is not very useful. Nevertheless we expect that a further optimization of the sensor structure, e.g. by means of a reduction of the air gap as well as the electret thickness by using $\mathrm{SiO}_{2}$ as the electret instead of Teflon-FEP, may yield a reliable sensor that especially can be applied in dynamic measurements.

\section{CONCLUSIONS}

W $\mathrm{E}$ have shown that integration of $\mathrm{SiO}_{2}$ or Teflon electrets yield small sensors which may have rather good sensor properties. The processing required for the realization of these sensors has been presented and their performance has been illustrated by means of some experimental results.

The results of the pressure sensor are rather disappointing, mainly due to the low value of the pressure sensitivity in relation to a considerable temperature sensitivity. An improvement of the pressure sensitivity or a reduction of the temperature sensitivity seems not realistic or not sufficient. The increased temperature sensitivity for the pressure sensor as compared to the microphone, is mainly due to the fact that in a pressure sensor the frequency regions of pressure and temperature partly coincide, which is not the case in a microphone. Therefore we conclude that electrets are not useful in the present PRESSFET realization or in other sensors for static or extremely low-frequency applications.

In the case of the microphone however, the theoretical and the experimental results are very promising. In this type of electret sensor we have exploited the micromachining possibilities of silicon and the electret properties of $\mathrm{SiO}_{2}$. In this way we have realized sensors with high sensitivity and good frequency response.

\section{REFERENCES}

[1] K. E. Peterson, "Silicon as a mechanical material", Proc. IEEE, Vol. 70, pp. 420-457, 1982.

[2] J. A. Voorthuyzen, A. J. Sprenkels, W. Olthuis and P. Bergueld, "Research and development on miniaturized electrets", IEEE Trans. Electr. Ins., Vol. 24, (this issue), 1989.

[3] A. J. Sprenkels, A silicon subminiature electret microphone, Thesis, Twente University, 1988.

[4] G. M Sessler, "Topics in applied physics", Vol. 33, Electrets, Springer-Verlag, Berlin, 1980.

[5] J. A. Voorthuyzen and P. Bergueld, "The PRESSFET, an integrated electret-MOSFET based pressure sensor", Sensors and Actuators, Vol. 14, pp. $349-360,1988$.

Manuscript was received on 17 Feb 1989, in revised form 6 Mar 1989. 